\title{
Molecular analysis of phylogeographic subspecies in three Ponto-Caspian sturgeon species
}

\author{
Andreea Dudu, Sergiu Emil Georgescu and Marieta Costache \\ Department of Biochemistry and Molecular Biology, Faculty of Biology, University of Bucharest, Bucharest, \\ Romania.
}

\begin{abstract}
Sturgeons (Order Acipenseriformes) represent an extremely valuable natural resource that is now facing depletion. In the current study we evaluate if the traditional classification in subspecies of Acipenser gueldenstaedtii, Acipenser stellatus and Huso huso, endemic to Ponto-Caspian region is sustained by molecular analysis and if these represent Evolutionary Significant Units (ESUs) that should be managed separately in conservation programs. To examine the classification of taxonomic entities we sequenced a fragment of the mitochondrial control region in case of three sturgeon species that inhabit the North-western of Black Sea and migrate for reproduction in the Lower Danube. Beside these sequences, we used previously published sequences from sturgeon individuals sampled in the Black Sea, Azov Sea and Caspian Sea. We determined the genetic diversity and genetic differentiation, conducted a Population Aggregation Analysis (PAA) and inferred an intraspecific molecular phylogeny and haplotype network. The results indicated a low level of genetic differentiation between the geographically designated subspecies and did not support a significant divergence or reciprocal monophyly between them. Our results confirm previous genetic studies with smaller samples sizes, but additional analyses including nuclear markers should be conducted for proper recommendations aiming at the development of conservation programs.
\end{abstract}

Keywords: Ponto-Caspian sturgeon, subspecies, ESUs, mitochondrial markers.

Received: January 15, 2014; Accepted: May 24, 2014.

\section{Introduction}

Sturgeons represent an ancient group of fish with slow evolution, which maintained their morphological characteristics for more than 200 million years (Bemis and Kynard, 1997). Overfishing and poaching for the valuable caviar and meat, the interruption of the migratory routes as a consequence of dam constructions and pollution represents the factors that have affected sturgeon populations worldwide.

At present, 27 species of sturgeon and paddlefishes (Order Acipenseriformes) are found on the IUCN Red List, the majority being classified as "Critically Endangered", and four species are now possibly extinct (http://www.iucn.org). In this situation it is crucially important to develop efficient programs aiming at conservation, repopulation of their original habitats and aquaculture, not only for supplying the request of the market for meat and caviar, but also to raise appropriate reproducer sturgeons in the attempt of recover wild populations. A sustained effort must now to be directed at protecting the

Send correspondence to Sergiu Emil Georgescu. Department of Biochemistry and Molecular Biology, Faculty of Biology, University of Bucharest, Splaiul Independentei 91-95, 050095 Bucharest 5, Romania. E-mail: georgescu_se@yahoo.com. diversity residing within species by altering harvest and hatchery release programs to sustain locally adapted gene pools (Doukakis et al., 1999).

In order to have efficient conservation and restocking programs it is absolutely necessary to designate and properly manage conservation units. The diagnosis of conservation units typically includes recognition of genetic diversity and specifically unique evolutionary lineages or Evolutionary Significant Units (ESUs) (Blair et al., 2013). In fact one of the aims of endangered species conservation should be directed to the identification of ESUs, defined broadly as a subspecies, population or group of populations with a high genetic and ecological distinctiveness (Allendorf and Luikart, 2007) and characterized by reproductive isolation and adaptation (Waples, 1991); reciprocal monophyly at mtDNA loci (Moritz, 1994); the lack of "ecological exchangeability" (different life histories, morphology, quantitative trait locus variation, habitat, predators, etc.) and of "genetic exchangeability" (no recent gene flow, concordance between phylogenetic and geographic discontinuities) (Crandall et al., 2000).

Historically, the Ponto-Caspian region hosted numerous sturgeon species, but nowadays only six of them inhabit the seas from the region and their tributaries (Secor et al., 2000). The Ponto-Caspian region comprises the basins of 
the Black Sea, Caspian Sea and Azov Sea. The Black Sea and Azov Sea are interconnected by Kerch Strait, while the Caspian Sea is completely separated from the other two basins. The major rivers in this region are Volga and Ural (flowing into the Caspian Sea), Danube, Dnieper and Dniester (flowing into the Black Sea), Don and Kuban (flowing into the Azov Sea), which together with their tributaries provide spawning grounds for anadromous sturgeon species from the three seas.

Based on morphological and ecological features there is a subspecies designation that corresponds to their distinct geographical distribution into separate basins: H. huso maeoticus is originating in the Azov Sea, H. huso ponticus in the Black Sea and H. huso caspicus in the Caspian Sea; A. stellatus donensis is specific to the Azov Sea, A. stellatus ponticus to the Black Sea and A. stellatus stellatus to the Caspian Sea (Pirogovskii et al., 1989; Shubina et al., 1989). Within A. gueldenstaedtii, one subspecies corresponds to the Black Sea/Azov Sea (A. gueldenstaedtii colchicus) and one to the Caspian Sea (A. gueldenstaedtii gueldenstaedtii) (Vlasenko et al., 1989).

The main goal of our study was to infer the conservation units for three anadromous sturgeon species (H. huso, A. stellatus and A. gueldenstaedtii) from the Ponto-Caspian region by using the mitochondrial control region as marker to clarify the intraspecific genetic differentiation of these species. By using sequences from the GenBank data base for sturgeons from the Caspian Sea and Azov Sea, as well as our own sequences for sturgeons individuals sampled in the Lower Danube as references for the Black Sea population, we tested phylogeographic structure of the three sturgeon species and the genetic support and validity of their classification as subspecies and distinctive units for conservation.

\section{Material and Methods}

\section{Sampling and DNA isolation}

The individuals used in this study were captured in the Lower Danube in a national scientific research study for restocking and monitoring. Sampling did not jeopardize the life and welfare of the animals as only very small fragments of fins were sampled before the animals were tagged and again released into the river.

Biological samples represented by small fin tissue pieces were collected between 2001 and 2008 from 78 sturgeon individuals (27 A. stellatus, 28 A. gueldenstaedtii and 23 H. huso) from the Lower Danube River. The provenance of the sturgeon samples used in this study is shown in Figure 1.

On field collection, the tissue specimens were fixed in 96\% ethanol and preserved as such until the DNA extraction was accomplished. Total DNA was isolated using a standard phenol-chloroform method (Taggart et al., 1992) with minor modifications.

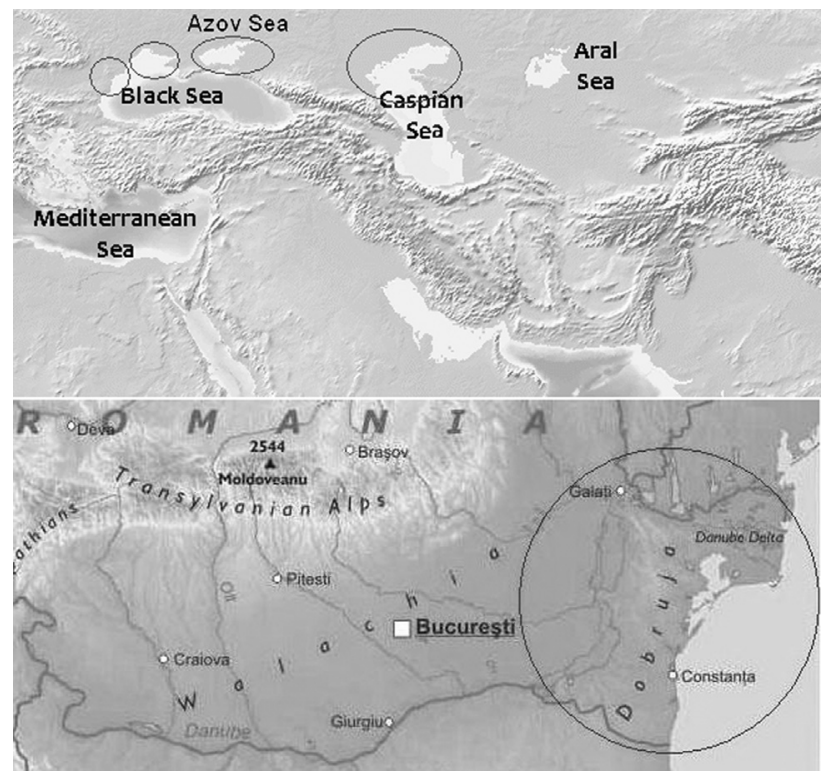

Figure 1 - Sampling region for Romania and the provenance of samples obtained from GenBank.

\section{Mitochondrial DNA analysis}

The entire control region and partial sequences of the flanking tRNA genes were amplified using the primer pair ProF: 5'-ACCCTTAACTCCCAAAG-3' and PheR: 5'-GCTTTAGTTAAGCTACGC-3'. The primers used are matching to proline and phenylalanine tRNA genes, respectively. The primers were designed with Primer 3 software (Koressaar and Remm, 2007; Untergrasser et al., 2012).

The PCR amplifications were conducted in a thermocycler GeneAmp 9700 PCR System (Applied Biosystems) under the following conditions: $95^{\circ} \mathrm{C}$ for $10 \mathrm{~min}$, followed by 35 cycles of $95^{\circ} \mathrm{C}$ for $30 \mathrm{~s}, 52^{\circ} \mathrm{C}$ for $30 \mathrm{~s}$ and $72{ }^{\circ} \mathrm{C}$ for $60 \mathrm{~s}$. The reactions were carried out in $25 \mu \mathrm{L}$ final volume containing 50 ng DNA template, 1X AmpliTaq Gold Reaction Buffer, $1.5 \mathrm{mM} \mathrm{MgCl} 2,200 \mu \mathrm{M}$ of each dNTPs, $0.4 \mu \mathrm{M}$ of each primer and $0.5 \mathrm{U}$ of AmpliTaq Gold Polymerase (Applied Biosystems). The PCR products were purified using a WizardSV Gel and PCR Clean-Up System Kit (Promega).

Sequencing reactions were performed using the BigDye Terminator v3.1 Cycle Sequencing Kit (Applied Biosystems) and the purification of the sequencing products was completed with BigDye-XTerminator (Applied Biosystems). The purified fluorescently labeled products were loaded and analyzed into an ABI Prism 3130 Genetic Analyzer (Applied Biosystems). For excluding errors, both strands of the amplicons were sequenced.

\section{Data analysis}

The sequences obtained for the Lower Danube individuals were included in the group of sequences for the 
Black Sea sturgeon population and were aligned and compared with similar sequences from GenBank for sturgeons from the Black Sea, Azov Sea and Caspian Sea (see Table S1). The D-loop sequences were truncated to $731 \mathrm{bp}$ in A. stellatus, $641 \mathrm{bp}$ in $9 \mathrm{H}$. huso and $695 \mathrm{bp}$ in $A$. gueldenstaedtii to accommodate them to the shorter sequences from GenBank selected for analysis. For our sequences, the distinct haplotypes only were considered for further analyses. The sequences representing distinct haplotypes were deposited in GenBank with the following access numbers: KF431840 - KF 431846 (H. huso), KF431847 - KF431850 (A. gueldenstaedtii) and KF431851 - KF431857 (A. stellatus). The sequences were aligned using ClustalX 2.0.9 software (Larkin et al., 2007). The genetic diversity in terms of number of haplotypes, nucleotide diversity and haplotype diversity were calculated using DnaSP v5.1 (Librado and Rozas, 2009) The genetic differentiation between populations based on the fixation index $\left(\mathrm{F}_{\mathrm{ST}}\right)$ and other statistical indices of differentiation $\left(\right.$ Gamma $_{\mathrm{ST}}, \mathrm{H}_{\mathrm{s}}, \mathrm{K}_{\mathrm{s}}^{*}$ ) were computed using the same software. Statistical significance was tested by permutation with 10,000 replicates. The analysis was accomplished with gaps included and considered as a fifth state. The $\mathrm{N}_{\mathrm{m}}$ value calculated in DnaSP was used to estimate the gene flow based on $\mathrm{F}_{\mathrm{ST}}$. For this analysis we eliminated the two sequences of $H$. huso maeoticus because a larger number of samples would have been necessary for a precise diagnosis of subspecies definition and for inferring ESUs (Walsh, 2000).

The mtDNA sequences were analyzed by using the discrete character-based methodology of population aggregation analysis (PAA) (Davis and Nixon, 1992). The PAA groups taxa together based on the presence of fixed traits such that they are diagnosable distinct from one another (Cracraft, 1983).

We inferred a haplotype network of D-loop sequences using a median-joining algorithm implemented in NETWORK v4.6.1 (Bandelt et al., 1999) with default settings $(\varepsilon=0)$ and the variable sites weighted equally (weight $=10)$. The network was rooted with Acipenser baerii.

The phylogenetic relationships were inferred by Neighbor Joining (NJ) and Maximum Likelihood (ML) algorithms implemented in MEGA v5 (Tamura et al., 2011). The best-fit model of sequence evolution was selected using the Akaike Information Criterion (AIC) from ModelTest (Posada and Crandall, 1998). Bootstrap analyses (1000 replications) were used to assess the confidence of each node. The phylogenetic trees were visualized with TreeView (Page, 1996). The Siberian sturgeon, A. baerii (NC_017603) was used as an outgroup species both for network and tree construction.

\section{Results}

\section{Polymorphism analysis of mtDNA data}

Among the sequences that we obtained for individual sturgeons sampled in the Lower Danube River we identified four distinct haplotypes in 28 specimens of $A$. gueldenstaedtii, seven haplotypes in 27 specimens of $A$. stellatus, and seven haplotypes in 23 specimens of H. huso. For testing the subspecies existence hypothesis and for an overview of the genetic differentiation our sequences representing distinct haplotypes were aligned and compared with similar control region sequences from GenBank, corresponding to sturgeon from the Black Sea, Caspian Sea and Azov Sea. The sequence alignments comprised 32 D-loop sequences for A. gueldenstaedtii, 78 sequences for A. stellatus and 43 sequences for $H$. huso. Due to the reduced number of sequences deposited in GenBank for $H$. huso maeoticus originating in the Azov Sea, these were excluded from most further analyses, except for the network and tree constructions. In the alignments, gaps were included and considered as a fifth state. We identified 23 distinct haplotypes in A. gueldenstaedtii, 65 haplotypes in A. stellatus and 30 haplotypes in $H$. huso for sturgeon individuals with distinct geographical origin.

Among the 23 distinct haplotypes identified in $A$. gueldenstaedtii, five are shared by more than one individual. Thus, one haplotype was common to six individuals from the Black Sea population and one was shared by two individuals from the same population; one haplotype was shared by two individuals from the Azov Sea population, and each of other two haplotypes were common for two individuals sampled in the Caspian Sea basin. The sequences obtained for our samples of $A$. gueldenstaedtii from the Danube River represent unique haplotypes.

In the case of $A$. stellatus, ten of the 65 haplotypes were shared by multiple individuals and the others were unique haplotypes. Except for two haplotypes that were common for A. stellatus individuals belonging to different populations, the other haplotypes were shared by individuals from the same basin. The haplotypes for the A. stellatus sampled in the Danube River and belonging to the Black Sea population represent unique haplotypes, with one exception, when a haplotype was shared by one individual from the Black Sea population and one individual from the Caspian Sea population.

Among the 36 haplotypes identified in $H$. huso, 32 represented unique haplotypes, whereas the rest was shared by different individuals. Two of the seven haplotypes characteristic for $H$. huso individuals sampled in the Lower Danube were in common with some sequences from GenBank. Thus, one of the haplotypes was shared with one individual from the Black Sea population and two individuals from the Caspian Sea population, and the other one was shared with one individual from the Black Sea population. Table 1 presents the calculated values for diversity indices, such as haplotype 


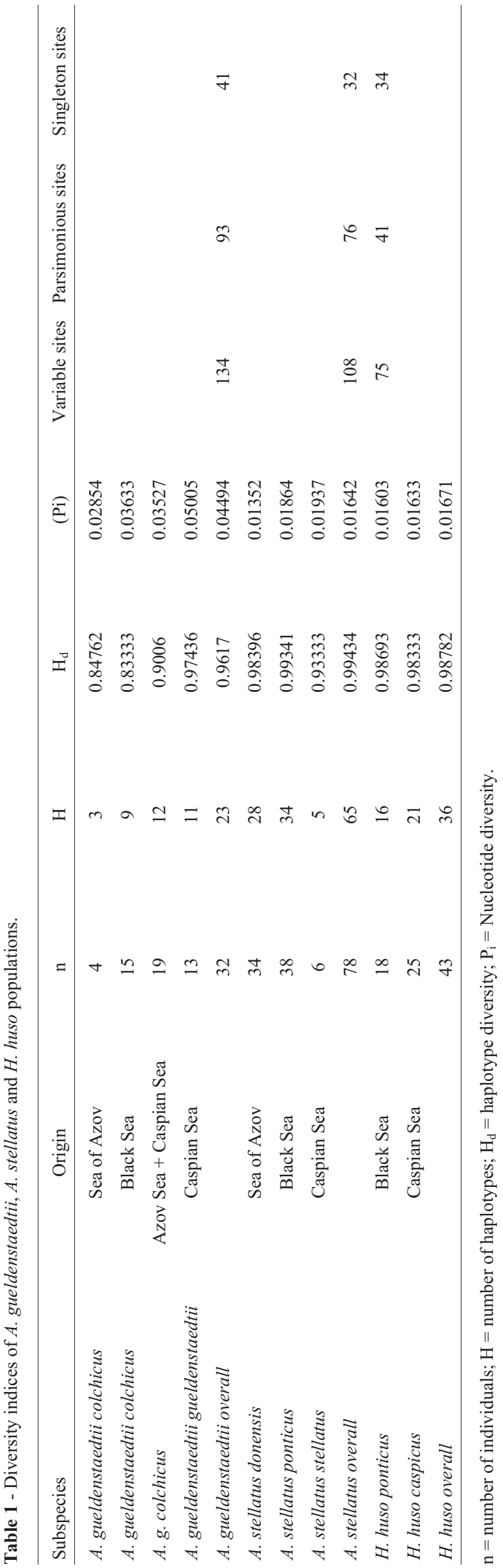

diversity, nucleotide diversity and number of polymorphic sites that describe the DNA sequence polymorphism and, thus, the amount of intraspecific genetic variation.

The haplotype diversity $\left(\mathrm{H}_{\mathrm{d}}\right)$ highlights the uniqueness of a particular haplotype in a given population. For $A$. gueldenstaedtii populations the haplotype diversity ranged from 0.83333 to 0.97436 , with a mean of $\mathrm{H}_{\mathrm{d}}=0.9617$, for A. stellatus populations the values for $\mathrm{H}_{\mathrm{d}}$ ranged from 0.99341 to 0.93333 , with a mean of 0.99434 , and for $H$. huso populations we obtained a haplotype diversity of 0.98693 and 0.98333 , with a mean of 0.98782 . Haplotype diversity was found to be high in all the sturgeon species, with an intraspecific value over 0.95 , and also when calculated separately for each populations.

In contrast, the nucleotide diversity $\left(\mathrm{P}_{\mathrm{i}}\right)$ was relatively low for each population. The average number of nucleotide differences per site between two randomly selected sequences, or nucleotide diversity, was 0.01642 in $A$. stellatus populations and 0.04494 in A. gueldenstaedtii populations. An intermediate value of 0.01671 , but very similar to the $\mathrm{P}_{\mathrm{i}}$ of $A$. stellatus populations, was determined for $H$. huso populations. The highest nucleotide diversity was found in A. gueldenstaedtii populations, particularly in A. gueldenstaedtii gueldenstaedtii from the Caspian Sea. Lower nucleotide diversity was denoted for $A$. gueldenstaedtii colchicus from the Black Sea and Azov Sea basins, but we need to consider that for the Azov Sea population we analyzed only four individuals. For the $A$. stellatus and $H$. huso populations, the values for $\mathrm{P}_{\mathrm{i}}$ were similar and lower than 0.02 , with A. stellatus ponticus and A. stellatus stellatus presenting the highest nucleotide diversity. Thus, nucleotide diversity was relatively low for all analyzed sturgeon populations. The low diversity highlighted by our analysis might be the consequence of a relative recent divergence between different sturgeon populations, or be due to a severe population bottleneck during sturgeon evolution.

\section{Population Aggregation Analysis (PAA)}

In A. gueldenstaedtii, we identified 134 variable sites in which we detected 136 polymorphisms (130 transitions, 3 transversions and 3 indels). A total of 108 variable sites including 110 polymorphisms (100 transitions, 9 transversions and 1 indel) were detected in A. stellatus. Among the 75 variable sites in $H$. huso thes included 80 different polymorphisms with 68 transitions, 7 transversions and 5 indels.

The PAA analysis of unique haplotypes, as identified by DNAsp, did not recover fixed nucleotide differences (characters), and thus it was not possible to differentiate between characters and traits (polymorphic sites) in the sequence data sets of the three Ponto-Caspian species (Figures S1, S2 and S3). The existence of diagnostic characters representing an obvious evidence of phylogenetic distinctiveness, if common to all analyzed individuals 
within a hypothetic ESU (subspecies or allopatric population), was not revealed by our analysis. Thus, the genetic evidences did not support the classical subspecies designation for A. gueldenstaedtii, A. stellatus and H. huso.

\section{Genetic differentiation analysis}

The divergence between populations was estimated mainly by Wright's Fixation Index $\left(\mathrm{F}_{\mathrm{st}}\right)$, which can range from 0 (no genetic differentiation) to 1 (complete differentiation between population). For a more reliable interpretation of genetic differentiation data we also evaluated the Gamma $_{\text {st }}$ values, which represent an unbiased estimate of $\mathrm{F}_{\mathrm{st}}$ that corrects for errors associated with incomplete sampling of populations and is more suitable for mitochondrial haplotype data (Weir and Cockerham, 1984). Pairwise differences of the populations were calculated as Fst values, which can also be used to measure the short-term genetic distances between the populations (Reynolds et al., 1983). The values for all statistical indices were similar, indicating low to moderate genetic differentiation between the populations (Table 2).

The $\mathrm{F}_{\mathrm{ST}}$ values were lower than 0.1 for $A$. stellatus and $H$. huso, indicating a low genetic divergence between populations, despite their distinct geographic origin. $A$. stellatus and $H$. huso showed no structuring, and the studied populations appeared to belong to one panmictic population. In contrast, the pairwise $F_{\mathrm{ST}}$ values were relatively high and statistically significant, indicating a certain divergence in A. gueldenstaedtii populations. Thus, a moderate level of genetic differentiation was found among the populations of $A$. gueldenstaedtii. The lowest level of differentiation was evidenced among the Black Sea and Azov Sea populations, the other comparisons within $A$. gueldenstaedtii showing a value of $\mathrm{F}_{\mathrm{ST}}>1$. For testing the subspecies hypothesis we analyzed as distinct groups $A$. gueldenstaedtii colchicus (from Black Sea and Azov Sea) vs. A. gueldenstaedtii gueldenstaedtii (from Caspian Sea). In this case, the values of statistical indices also indicated a moderate differentiation between the two groups. With two exceptions, the values of $\mathrm{N}_{\mathrm{m}}$ calculated from $\mathrm{F}_{\mathrm{ST}}$ are, all higher than 4. A small degree of differentiation was, thus, directly observed, while an intensive gene flow between the analyzed populations is indirectly inferred.

\section{Phylogenetic analysis of mitochondrial data}

Based on the AIC criterion we selected the Tamura 3-Parameter as the best-fit model of nucleotide evolution in a gamma distribution of rates for both NJ and ML analyses. The haplotypes were distributed randomly along the both $\mathrm{NJ}$ and ML trees and were not associated with geographic origin (i.e. the proposed subspecies) . The phylogenetic analysis did not support the grouping of haplotypes by geographic origin and/or classical subspecies designation. No monophyletic groups corresponding to populations/ subspecies were recovered (Figures 2, 3 and 4).

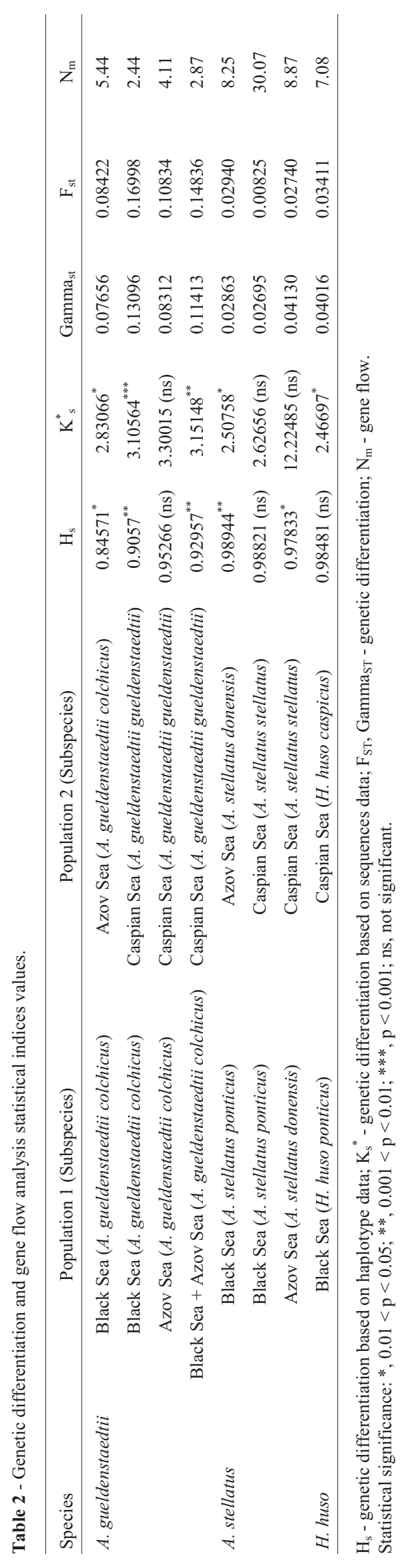


(a)
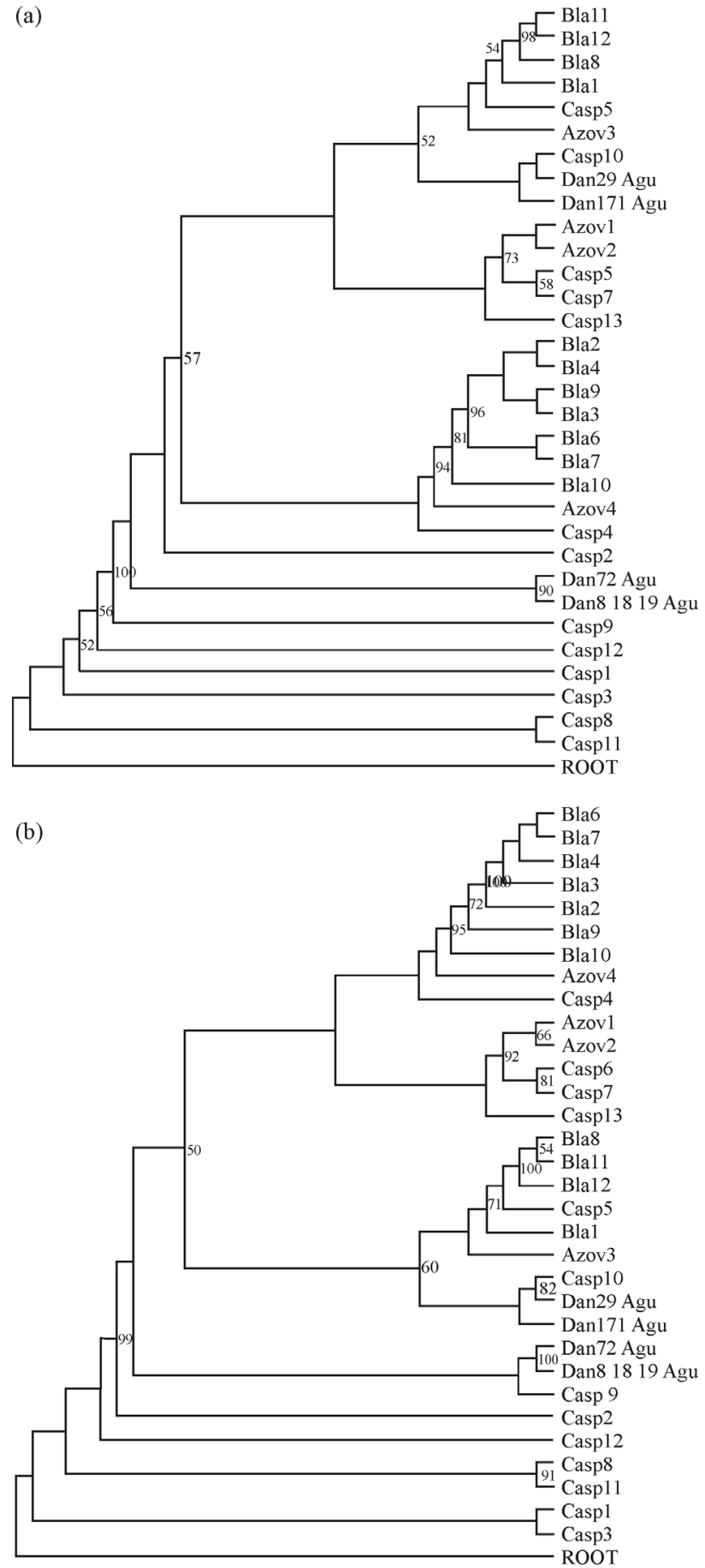

Figure 2 - Molecular phylogenetic trees inferred by partial D-loop sequences analysis by Neighbor-Joining (A) and Maximum Likelihood (B) in A. gueldenstaedtii constructed using the Tamura3 parameter plus $\mathrm{G}$ model with 1000 bootstrap iterations. ROOT indicates the outgroup species A. baerii (NC 017603). The numbers represent the confidence bootstrap values higher than 50. Bla= Black Sea; Casp $=$ Caspian Sea; Azov $=$ Azov Sea.

\section{mtDNA haplotype networks}

The haplotype networks of control region sequences showed the genealogical relationships among the haplo- types of three Ponto-Caspian sturgeon species (Figure 5). The haplotype networks did not reflect the results of the diversity indices and phylogenetic trees presented above. The shape of the networks indicated a complex structure with many median vectors (full black circles) and the majority of the circles had the characteristics of old haplotypes (low frequeny, restricted to one basin, connected by multiple mutational steps). Also, only few haplotypes were shared between the seas.

\section{Discussion}

For the three Ponto-Caspian sturgeon species a taxonomic classification in subspecies based on morphological, meristic and ecological traits and related to the water body in which they are found was already proposed (Pirogovskii et al., 1989; Shubina et al., 1989; Vlasenko et al., 1989). Although this taxonomic classification is not unanimously accepted, confirmation might be very important for coordinating useful conservation programs aiming at identifying and managing the proper conservation units of these highly endangered species. A previous molecular study did not support the subspecies designation in sturgeons (Doukakis et al., 1999) and supported caveats regarding the classification of subspecies in A. stellatus. The differentiation between A. stellatus stellatus (Caspian Sea) and A. stellatus donensis (Azov Sea) was made based on several characteristics, including head proportions, anal and dorsal fin characters, different rates of growth and maturity (Chugunov and Chugunova ,1964; Tsvetnenko, 1993), but later it was found that these features modify with growth and feeding (Holcick and Jedlicka, 1994) and, in consequence, such taxonomic nomenclature should not be used without genetic support (Birstein and Bemis, 1997; Doukakis et al., 1999).

Following this advice, upon analyzing the mitochondrial control region, we did not find evidence for significant divergence and reciprocal monophyly among the sturgeon populations and, consequently, our results did not support their classification as subspecies. Our population aggregation analysis also did not recover any ESUs, although the confidence of our results would increase with a larger number of samples. According to Walsh (2000), a critical number of 59 individuals is necessary in order to detect hidden character states in a population. Unfortunately, in sturgeons acquiring a larger number of biological samples is very challenging given the drastically reduced population and the difficulty of sampling due to their particular life cycle and behavior. Also, the fact that the PAA analysis did not reveal any fixed character in subspecies does not exclude that the respective haplotypes are exclusive to a subspecies, or even more, that the subspecies exist as proposed.

All the three sturgeon species as a whole display a high degree of haplotype diversity. The values of haplotype for species diversity lie in the range of 0.96 to 0.99 , which is high when compared to many other species. The same pat- 
(a)

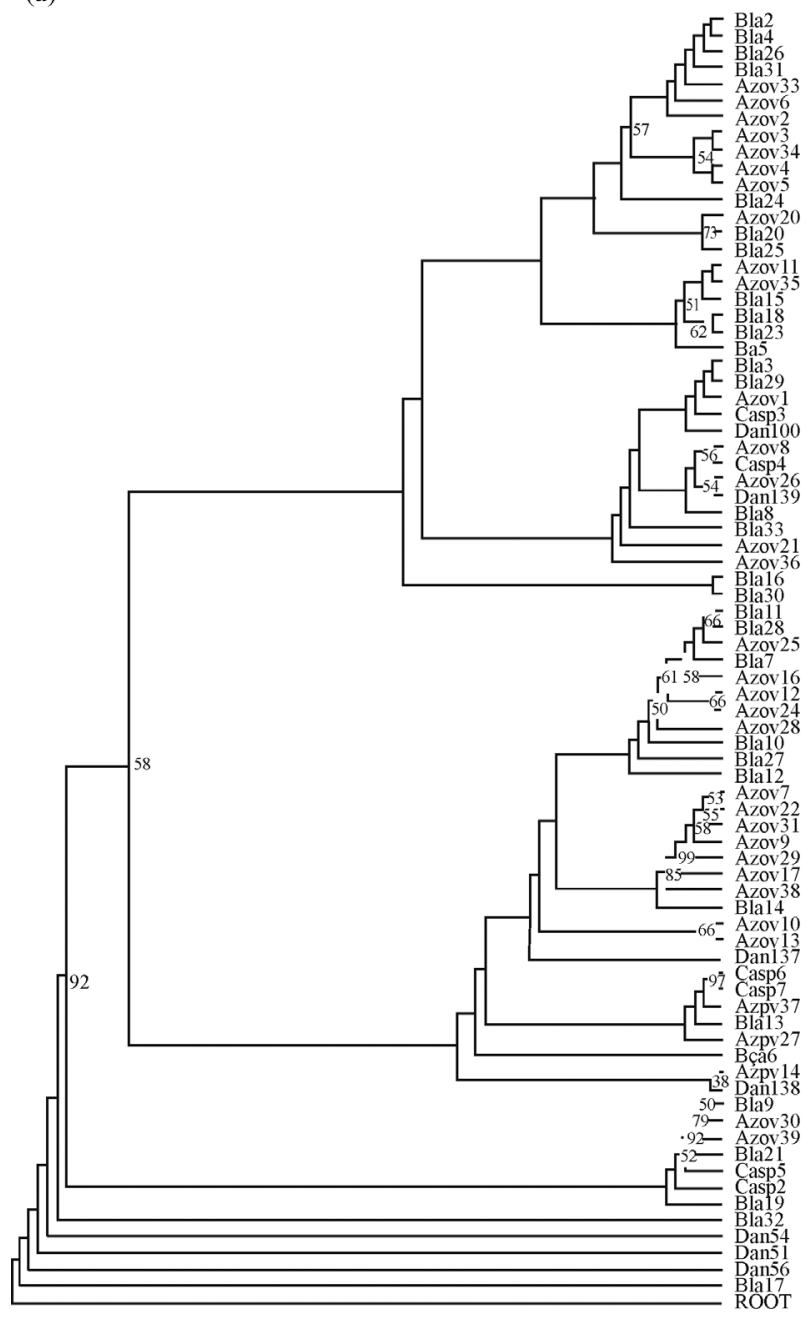

(b)

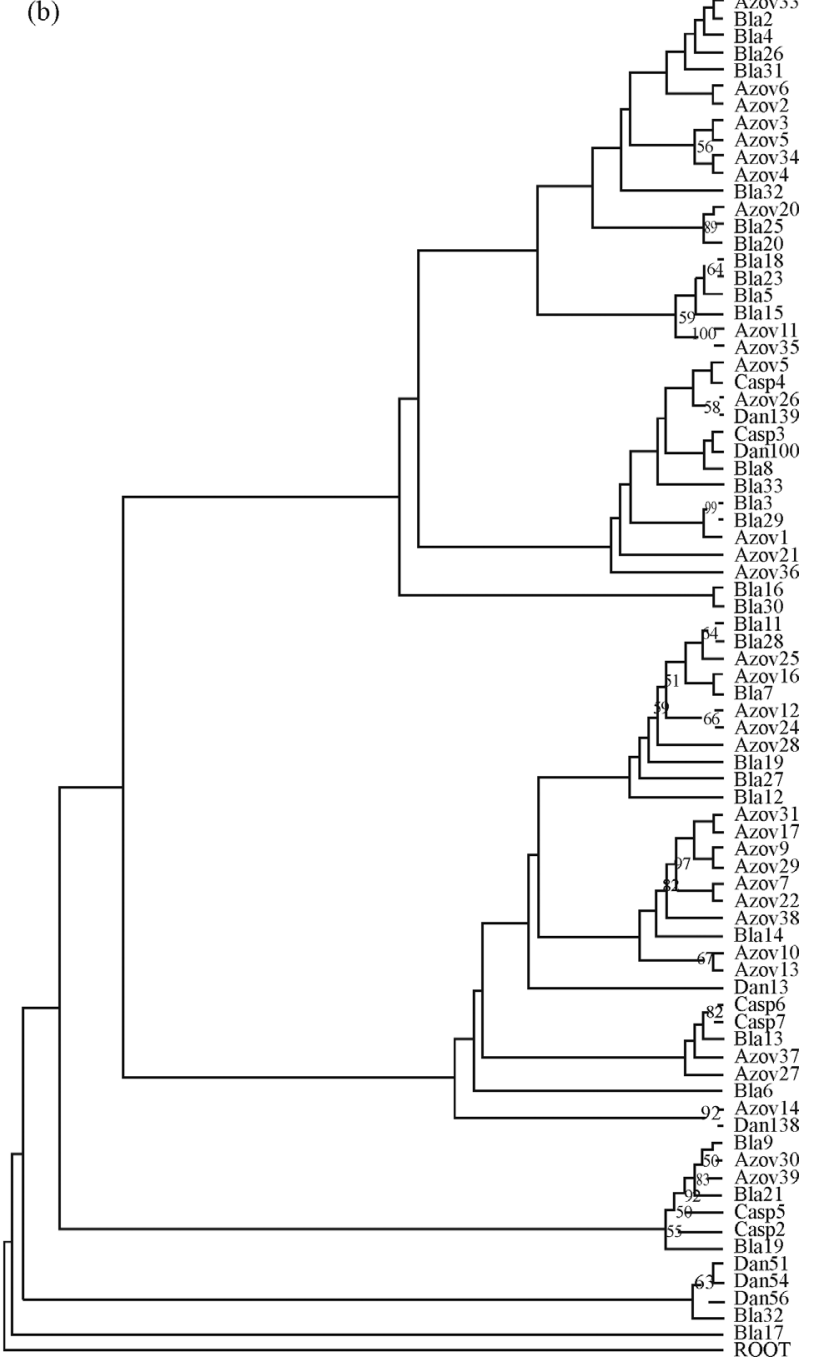

Figure 3 - Molecular phylogenetic trees inferred by partial D-loop sequences analysis by NJ (a) and ML (b) in A. stellatus constructed using Tamura3 parameter plus $\mathrm{G}$ model with 1000 bootstrap iterations. ROOT- indicates the outgroup species A. baerii (NC_017603). The numbers represent the confidence bootstrap values higher than 50. Bla= Black Sea; Casp = Caspian Sea; Azov = Azov Sea.

tern of diversity is evident for the investigated subspecies/ populations. Nucleotide diversity is considerate to be low to moderate for values from $0.01-0.04$. The highest nucleotide diversity $\left(\mathrm{P}_{\mathrm{i}}-0.04494\right)$ was found in $A$. gueldenstaedtii and was comparable to the value found by Khoshkholgh et al. (2011) in A. persicus from the South Caspian Sea, using as well the mitochondrial control region as molecular marker. Although nucleotide diversity is rather reduced (except for A. gueldenstaedtii) at the species, subspecies and population levels, the values obtained are significantly higher than those observed in other sturgeon species, like $A$. brevirostrum (Grunwald et al., 2002) and A. oxyrinchus oxyrinchus (Wirgin et al., 2000, 2005). Nonetheless, the congruence between these results should be treated with care, considering differences in the number of samples included in the studies and the length of the analyzed sequences.
Despite the wide distribution range and the presence of designated subspecies in isolated water bodies, our results show relatively little geographic differentiation and significant gene flow between the subspecies. A sufficient gene flow between populations can slow down or prevent the process of geographic differentiation and, thus, resulting in a small population structure over large areas.

The results illustrated by the phylogenetic trees are congruent with those of the genetic differentiation analysis. The results of the genetic differentiation and gene flow statistical analysis and the absence of monophyletic groups corresponding to subspecies/populations are arguments in favor of the questionable taxonomic classification. In contrast, the haplotype network results showed a strong population structure. The circles of the networks retain the attributes of old haplotypes, and only few haplotypes are shared between the seas basins. Thus, only two among 36 haplotypes of $H$. huso, none among 23 haplotypes of $A$. 
(a)

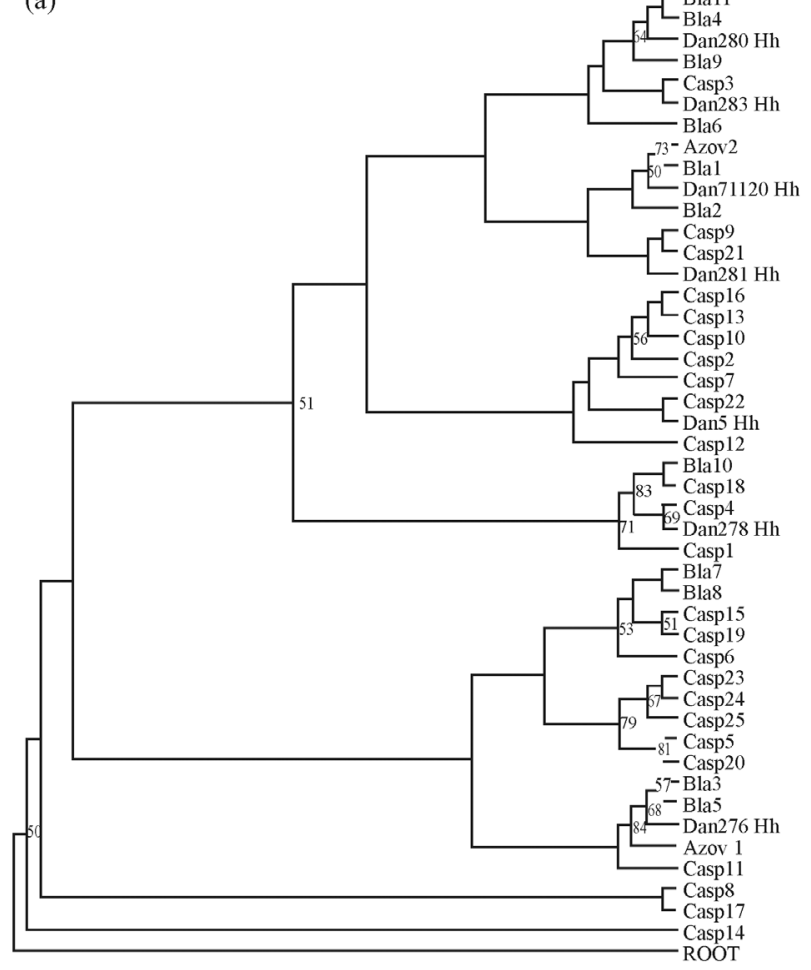

(b)

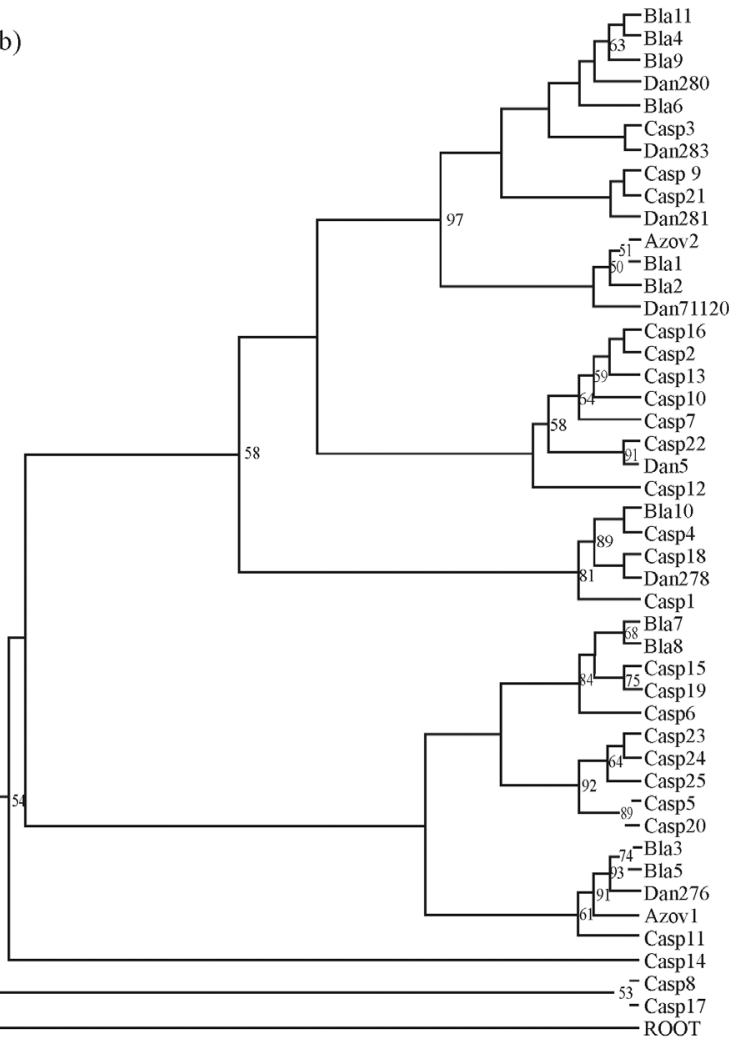

Figure 4 - Molecular phylogenetic trees inferred by partial D-loop sequences analysis by Neighbor-Joining (A) and Maximum Likelihood (B) in $H$. huso constructed using the Tamura3 parameter plus $\mathrm{G}$ model with 1000 bootstrap iterations. ROOT indicates the outgroup species $A$. baerii (NC_017603). The numbers represent the confidence bootstrap values higher than 50. Bla= Black Sea; Casp $=$ Caspian Sea; Azov $=$ Azov Sea
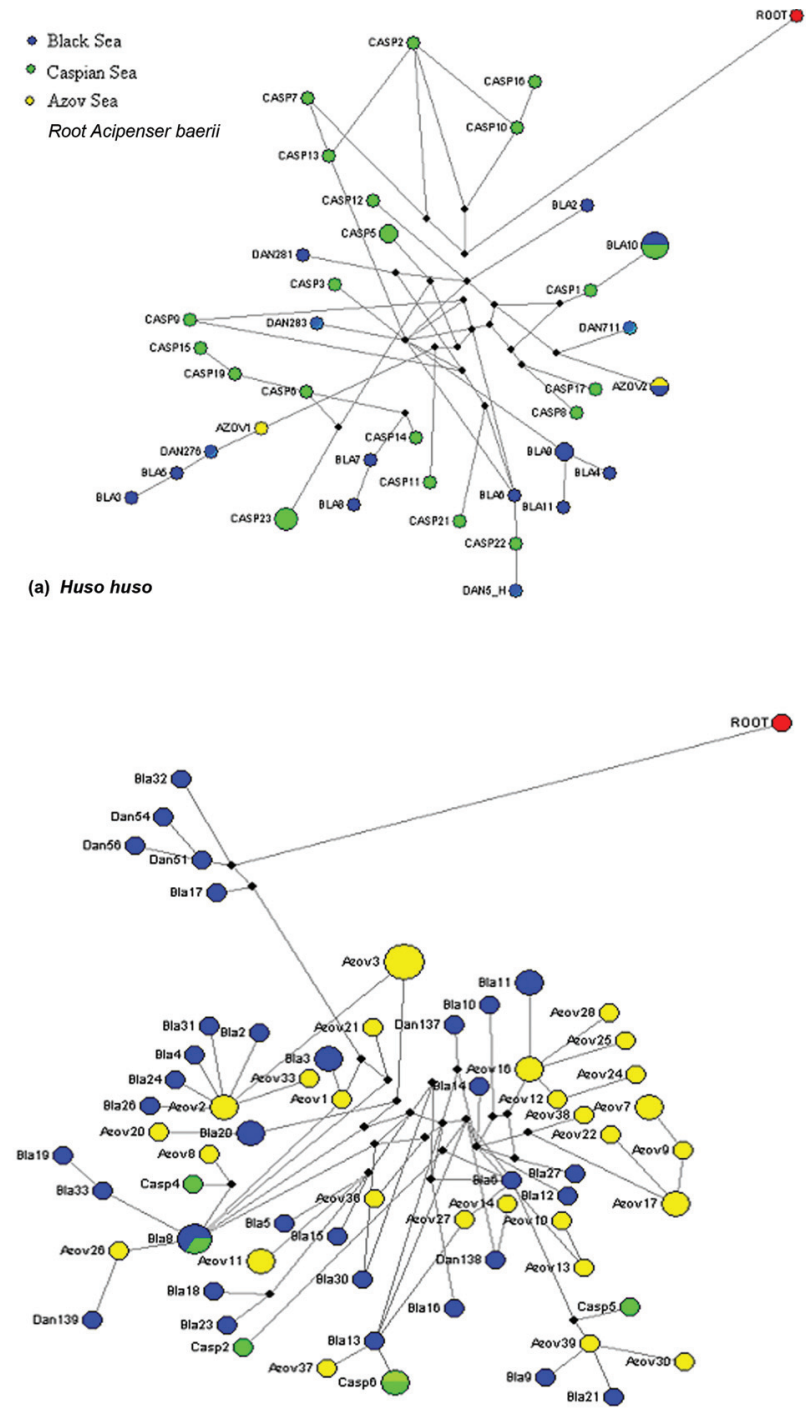

(b) Acipenser stellatus

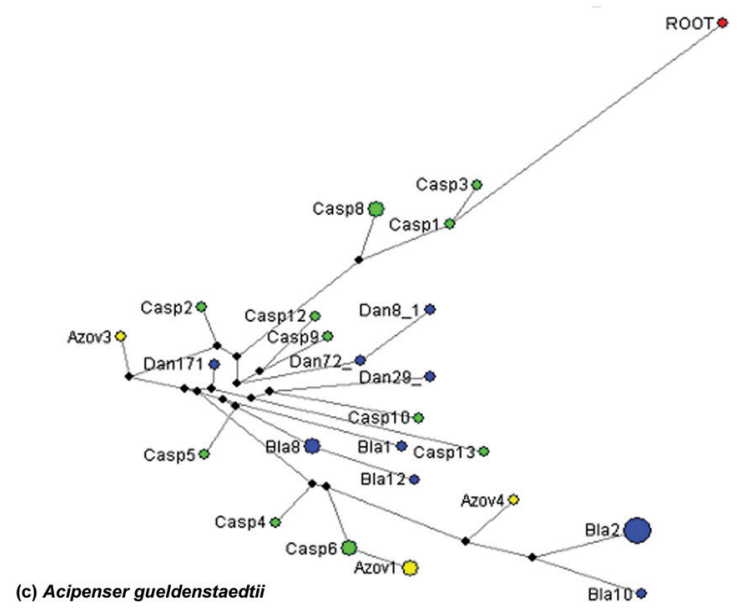

Figure 5 - Median-Joining networks for D-loop mtDNA haplotypes in (A) H. huso, (B) A. stellatus and (C) A. gueldenstaedtii populations. Circle size is proportional to haplotype frequency. Full black circles represent median vectors (missing haplotypes). Colors indicate the geographic origin of haplotypes. Branch lengths are proportional to the number of substitutions per nucleotide site. 
gueldenstaedtii and only one haplotype among 65 in $A$. stellatus are common to the different basins. Despite the fact that no star-like groupings were identified and the shape of the haplotypes networks might rather suggest a strong mixture of individuals, based on the previous arguments we consider that the networks reflect a strong population structure, even though this was revealed by the statistical indices.

A higher genetic structuring was found in $A$. gueldenstaedtii. This species exhibits great morphological variation, presenting multiple morphs and biological forms, including resident freshwater populations and spring and autumn migratory forms (Sokolov and Tspekin, 1996). Furthermore, within A. gueldenstaedtii genetically distinct lineages for undistinguishable morphology were inferred (Birstein et al., 2000).

The lack of differentiation and the considerable gene flow found in the analyzed sturgeon species could be the result of a relatively recent genetic exchange mediated through the geographical connection established between the three water bodies, but more probably it is the consequence of anthropic influence.

The origin of sturgeon species with a Ponto-Caspian distribution is probably related to the Tethys and Paratethys Seas. The Paratethys Sea was formed during the Late Jurassic (about $160 \mathrm{Mya}$ ) and was isolated during the Oligocene (about $34 \mathrm{Mya}$ ), when the connections with Tethys Sea were considerably reduced. The Paratethys Sea was localized 15 Mya within the current territory of the Black Sea, Azov Sea, Caspian Sea and Aral Sea (Jones and Simmons, 1996). A. gueldenstaedtii, or its ancestor, appeared in this period, while the rest of the species in the group appeared in the Late Miocene and Late Pleistocene as a result of global and local climate changes.

According to paleontological and molecular data, the dispersion of Ponto-Caspian sturgeon species in the Black Sea, Azov Sea and Caspian Sea occurred in the Pleistocene almost 1.5 Mya (Birstein and DeSalle, 1998). The Caspian Sea was the main refuge for the Ponto-Caspian species for the period 15-1.5 Mya. In this period, the anadromous sturgeons like A. gueldenstaedtii, A. stellatus and H. huso have probably migrated repeatedly from the Caspian Sea to the Black Sea and Azov Sea through water connections that were established between the three basins. Currently, the Azov Sea and the Black Sea are still connected through the Kerch Strait. In the light of evolutionary history of PontoCaspian sturgeon species, the small intraspecific differentiation might be a consequence of the genetic exchange mediated through the present water connections on a larger scale over 1.5 million years (Doukakis et al., 1999; Zubakov, 1988).

Similar results showing no or little genetic differentiation within species were found not only in sturgeons (Brown et al., 1993; Guenette et al., 1993), but also in salmon species. Based on phenotypic and ecological varia- tion, populations of anadromous Salmo trutta from the Black, Caspian and Aral Seas have been classified into distinct taxa and considered as different subspecies. The results of molecular studies based on allozyme and mitochondrial markers, however, provided only weak support for this taxonomic distinction (Bernatchez and Osinov, 1995; Osinov and Bernatchez, 1996). Moreover, no genetic differentiation was found between anadromous and resident forms of Salmo trutta, despite their morphological, demographical and ecological characteristics and the fact that have even been classified as different species in the past (Charles et al., 2005).

Another source of gene flow is anthropic intervention by the intensive restocking programs of tributaries with sturgeons raised in aquaculture conditions to support the declining wild populations from the Black, Caspian and Azov Seas. Since there is a severe decline of all these species, restocking measures are ongoing for all three basins. Currently it is considered that almost $100 \%$ of beluga in the Volga River is hatchery reared. There are data showing that there has also been an intensive restocking in the Caspian Sea, since that $91 \%$ of each generation in this species is estimated to come from hatchery stock (Khodorevskaya et al., 2009). For A. gueldenstaedtii reports indicate that in the early 1990 s, between $30-55 \%$ of the Caspian stock originated from hatcheries (Khodorevskaya et al., 2000). Also, according to some opinions there is no native spawning population remaining in the Azov Sea, but only stocked individuals (Gesner et al., 2010). In the early 1990s, it was estimated that nearly $100 \%$ of the Azov Sea population and $30 \%$ of the Caspian Sea population of A. stellatus were from stocking. More recent estimates are that more than $50 \%$ of the Caspian Sea populations are from stocking (Qiwei, 2010). The restocking programs implying the three Ponto-Caspian species are currently practiced by different countries, like Russia, Romania, Khazakstan, Azerbaidjan, Iran, etc.. In the former Soviet Union, the restocking programs started in the 1960s as a consequence of limitation of spawning grounds following the construction of dams (Secor et al., 2000; Chebanov et al., 2002; Doukakis et al., 2005). The Caspian basin has lost a considerable surface (almost $70 \%$ ) of spawning grounds mainly due to hydroelectric power stations. For example, only the Volgograd dam has dramatically decreased the area of spawning grounds in the Volga River, while the Don River dam removed 68,000 ha of spawning ground, and flow regulation in the Kuban led to the loss of 140,000 ha of spawning grounds (Khodorevskaya et al., 2009; Gesner et al., 2013).

Especially in the past, restocking was made without taking into consideration the origin of sturgeon genitors and without a genetic characterization of individuals. In this case, it is not unexpected that the genetic structure of populations was altered as a consequence of hatchery practices and stocking of individuals belonging to other basin. Thus, almost half of the sturgeons released annually from 
the five hatcheries in the Azov Sea were originated from the Caspian Sea population. Apparently, this was also a common practice for A. stellatus and A. gueldenstaedtii. For stellate sturgeon the introduction of fertilized eggs from the Caspian Sea into the Azov Sea affected the genetic integrity of Azov Sea population (Chebanov et al., 2002).

Our study based on mitochondrial control region analysis reveals a general high genetic diversity within the three Ponto-Caspian sturgeon species but except for $A$. gueldenstaedtii, only very low differentiation between populations. The results of the mtDNA analyses do not support the existence of distinct subspecies corresponding to different basins and reflect a heterogeneous genetic composition of populations, probably as a consequence of uncontrolled restocking practices. A study on sampless collected prior to the onset of restocking programs and now representing museum specimens could be very useful to establish if the lack of differentiation is the result of geological changes or of human intervention, but such a study appears to be very difficult to realize. No ESUs were inferred, limiting thus the recommendations for conservation. For a correct detection of appropriate management units, a larger number of samples should be included in the study, but this might be very challenging considering the current status of sturgeon populations.

\section{Acknowledgments}

This work was supported by the PN-II-PT-PCCA Project 116/2012 "Genetic evaluation and monitoring of molecular and biotechnological factors that influence productive performance of Danube sturgeon species bred in intensive recirculating systems". D.A. was supported by the European Social Funding through the Sectorial Operational Programme for Human Resources Development POSDRU/159/1.5/S/133391.

\section{References}

Allendorf FW and Luikart G (2007) Conservation and the Genetics of Populations. Blackwell Publishing, Oxford, 624 pp.

Bandelt H-J, Forster P and Röhl A (1999) Median-joining networks for inferring intraspecific phylogenies. Mol Biol Evol 16:37-48.

Bemis WE and Kynard B (1997) Sturgeon rivers: An introduction to Acipenseriformes biogeography and life history. In: Birstein VJ, Waldman JR and Bemis WE (eds) Sturgeon Biodiversity and Conservation. Kluwer Academic Publishers, Dordrecht, pp 167-183.

Bernatchez L and Osinov AG (1995) Genetic diversity of trout (genus Salmo) from its most eastern native range based on mitochondrial DNA and nuclear gene variation. Mol Ecol 4:285-297.

Birstein VJ and Bemis WE (1997) How many species are there within the genus Acipenser? In: Birstein VJ, Waldman JR and Bemis WE (eds) Sturgeon Biodiversity and Conservation. Kluwer Academic Publishers, Dordrecht, pp 25-71.
Birstein VJ and DeSalle R (1998) Molecular phylogeny of Acipenserinae. Mol Phylogenet Evol 9:141-155.

Birstein VJ, Doukakis P and DeSalle R (2000) Polyphyly of mtDNA lineages in the Russian sturgeon, Acipenser gueldenstaedtii: Forensic and evolutionary implications. Conserv Genet 1:81-88.

Blair ME, Gutierrez-Espeleta GA and Melnick DJ (2013) Subspecies of the Central American squirrel monkey (Saimiri oerstedii) as units for conservation. Int J Primatol 34:86-98.

Brown JR, Beckenbach AT and Smith MJ (1993) Intraspecific DNA sequence variation of the mitochondrial control region of white sturgeon (Acipenser transmontanus). Mol Biol Evol 10:326-341.

Charles K, Guyomard R, Hoyheim B, Ombredane D and Bagliniere JL (2005) Lack of genetic differentiation between anadromous and resident sympatric brown trout (Salmo trutta) in a Normandy population. Aquat Living Resour 18:65-69.

Chebanov MS, Karnaukhov GI, Galich EV and Chmir YN (2002) Hatchery stock enhancement and conservation of sturgeon, with an emphasis on the Sea of Azov populations. J Appl Ichthyol 18:463-469.

Chugunov NL and Chugunova NI (1964) A comparative commercial and biological characteristics of the Sea of Azov acipenserids. Tr VNIRO 51:87-112.

Cracraft J (1983) Species concepts and speciation analysis. In: Richard F Johnston and Dennis M Power (eds) Current Ornithology, Vol 1. Plenum Press, New York, pp 159-187.

Crandall KA, Bininda-Emonds OR, Mace GM and Wayne RK (2000) Considering evolutionary processes in conservation biology. Trends Ecol Evol 15:290-295.

Davis JI and Nixon KC (1992) Populations, genetic variation and the delimitation of phylogenetic species. Syst Biol 41:421435.

Doukakis P, Birstein VJ and DeSalle R (2005) Intraspecific structure within three caviar-producing sturgeons (Acipenser gueldenstaedtii, A. stellatus, and Huso huso) based on mitochondrial DNA analysis. J Appl Ichthyol 21:457-460.

Doukakis P, Birstein VJ, Ruban GI and Desalle R (1999) Molecular genetic analysis among subspecies of two Eurasian sturgeon species Acipenser baerii and A. stellatus. Mol Ecol 8:S117-S127.

Grunwald C, Stablie J, Waldman JR, Gross R and Wirgin I (2002) Population genetics of shortnose sturgeon Acipenser brevirostrum based on mitochondrial DNA control region sequences. Mol Ecol 11:1885-1889.

Guenette S, Fortin R and Rassart E (1993) Mitochondrial DNA variation in lake sturgeon (Acipenser fulvescens) from the $\mathrm{St}$ Lawrence River and James Bay drainage basins in Quebec, Canada. Can J Fish Aquat Sci 50:659-664.

Holcick J and Jedlicka L (1994) Geographical variation of some taxonomically important characters in fishes: The case of bitterling Rodheus sericeus. Environ Biol Fish 41:147-170.

Jones RW and Simmons MD (1996) A review of the stratigraphy of Eastern Paratethys (Oligocene-Holocene). Bull Br Mus Nat Hist Geol 52:25-49.

Khodorevskaya RP, Krasikov EV, Dovgopol GF and Zhuravleva OL (2000) Formation of the stock of Caspian Acipenserids under present-day conditions. J Ichthyol 40:602-609.

Khodorevskaya RP, Ruban GI and Pavlov DS (2009) Behaviour, Migrations, Distribution and Stocks of Sturgeons in the 
Volga-Caspian Basin. Books on Demand $\mathrm{GmbH}$, Norderstedt, $233 \mathrm{pp}$.

Khoshkholgh M, Pourkazemi M, Nazari S and AzizzadehPormehr L (2011) Genetic diversity in the Persian sturgeon, Acipenser percicus, from the south Caspian Sea based on mitochondrial DNA sequences of the control region. Caspian J Environ Sci 9:17-25.

Koressaar T and Remm M (2007) Enhancements and modifications of primer design program Primer3. Bioinformatics 23:1289-1291.

Larkin MA, Blackshields G, Brown NP, Chenna R, McGettigan PA, McWilliam H, Valentin F, Wallace IM, Wilm A, Lopez $\mathrm{R}$, et al. (2007) Clustal W and Clustal X version 2.0. Bioinformatics 23:2947-2948.

Librado P and Rozas J (2009) DnaSPv5: A software for comprehensive analysis of DNA polymorphism data. Bioinformatics 25:1451-1452.

Moritz C (1994) Defining "evolutionary significant units" for conservation. Trends Ecol Evol 9:373-375.

Osinov A and Bernatchez L (1996) Atlantic and Danubian phylogenetic groupings of brown trout (Salmo trutta L.) complex: Genetic divergence, evolution, and conservation. J Ichthyol 36:762-786.

Page RD (1996) TreeView: An application to display phylogenetic trees on personal computers. CABiOS 12:357-368.

Pirogovskii MI, Sokolov LI and Vasil'ev V (1989) General introduction to fishes, Acipenseriformes. Huso huso Linnaeus, 1758. In: Holcik J (eds) The Freshwater Fishes of Europe. Vol. 1, Part II. AULA-Verlag, Wiesbaden, pp 156-200.

Posada D and Crandall KA (1998) Modeltest: Testing the model of DNA substitution. Bioinformatics 14:817-818.

Reynolds J, Weir BS and Cockerham CC (1983) Estimation of the coancestry coefficient: Basis for a short-term genetic distance. Genetics 105:767-779.

Secor DH, Arefjev V, Nikolaev A and Sharov A (2000) Restoration of sturgeons; lessons from the Caspian Sea sturgeon Ranching Programme. Fish Fish 1:215-230.

Shubina TN, Popova AA and Vasilev VP (1989) General Introduction to Fishes, Acipenseriformes. Acipenser stellatus Pallas, 1771. In: Holcik J (ed) The Freshwater Fishes of Europe. Vol. 1, Part II. AULA-Verlag, Wiesbaden, pp 395443.

Sokolov LI and Tspekin EA (1996) Acipenserids of the AzovBlack Sea and the Caspian basins (a historical review). Vopr Ikhtiol 36:15-27.

Taggart JB, Hynes RA, Prodohol PA and Ferguson A (1992) A simplified protocol for routine total DNA isolation from salmonid fishes. J Fish Biol 40:963-965.

Tamura K, Peterson D, Peterson N, Stecher G, Nei M and Kumar S (2011) MEGA5: Molecular Evolutionary Genetics Analysis using maximum likelihood, evolutionary distance, and maximum parsimony methods. Mol Biol Evol 28:27312739.

Tsvetnenko YB (1993) The effectiveness and genetic consequences of the introduction of the stellate sturgeon, Acipenser stellatus, into the Azov basin from the Caspian Sea. J Ichthyol 33:1-10.

Untergrasser A, Cutcutache I, Koressaar T, Ye J, Faircloth BC, Remm M and Rozen SG (2012) Primer3 - New capabilities and interfaces. Nucleic Acids Res 40:e115.
Vlasenko AD, Pavlov AV and Vasil'ev VP (1989) General Introduction to fishes, Acipenseriformes. Acipenser gueldenstaedtii Brandt, 1833. In: Holcik J (ed) The Freshwater Fishes of Europe. Vol. 1, Part II. AULA-Verlag, Wiesbaden, pp 294-344.

Walsh PD (2000) Sample size for diagnosis of conservation units. Conserv Biol 14:1533-1537.

Waples RS (1991) Definition of "species" under the Endangered Species Act: Application to Pacific Salmon. NOAA (National Oceanic and Atmospheric Administration). Technical Memorandum, National Marine Fisheries Service, F/NWC-194. Northwest Fisheries Science Center, Seattle.

Weir B and Cockerham C (1984) Estimating F statistics for the analysis of population structure. Evolution 38:1358-1370.

Wirgin I, Grunwald C, Carlson E, Stabile J, Peterson D and Waldman J (2005) Range-wide population structure of shortnose sturgeon Acipenser brevirostrum based on sequence analysis of mitochondrial DNA control region. Estuaries 28:406-421.

Wirgin I, Waldman JR, Rosko L, Gross R, Collins MR, Rogers SG and Stabile J (2000) Genetic structure of Atlantic sturgeon populations based on mitochondrial DNA control regions sequences. Trans American Fish Soc 129:476-486.

Zubakov VA (1988) Climatostratigraphic scheme of the Black Sea Pleistocene and its correlation with the oxygen-isotope scale and glacial events. Quart Res 29:1-24.

\section{Internet Resources}

Gesner J, Freyhof J and Kottelat M (2010) Acipenser gueldenstaedtii. In: IUCN 2013. IUCN Red List of Threatened Species. Version 2013.1, http://www.iucnredlist.org (accessed at September 14, 2013).

Qiwei W (2010) Acipenser stellatus. In: IUCN 2013. IUCN Red List of Threatened Species. Version 2013.1, http://www.iucnredlist.org (accessed at September 14, 2013).

NETWORK v4.6.1, http://www.fluxus-engineering.com/sharenet.htm (accessed at February 19, 2013).

\section{Supplementary Material}

The following online material is available for this article: - Table S1 - GenBank D-loop sequences from sturgeons subspecies.

- Figure S1 - Variable sites in unique haplotypes of $A$. gueldenstaedtii D-loop region.

- Figure S2 - Variable sites in unique haplotypes of $A$. stellatus D-loop region.

- Figure S3 - Variable sites in unique haplotypes of H. huso D-loop region.

This material is available as part of the online article from http://www.scielo.br/gmb.

Associate Editor: Antonio Solé-Cava

License information: This is an open-access article distributed under the terms of the Creative Commons Attribution License, which permits unrestricted use, distribution, and reproduction in any medium, provided the original work is properly cited. 